\title{
The Modal Analysis of Rotor-Rolling Bearing-Base System
}

$$
\text { Xuejun } \mathrm{Li}^{1, \mathrm{a}} \text { and Baihui } \mathrm{Yao}^{2, \mathrm{~b}}
$$

${ }^{1,2}$ Hunan Provincial Key Laboratory of Health Maintenance for Mechanical Equipment , Hunan University of Science and Technology, Xiangtan 411201, Hunan, China.

\author{
1'hnkjdxlxj@163.com, ²marta.yao@163.com
}

\section{Keywords: Modal Analysis; Rolling Bearing; Base; ANSYS}

\begin{abstract}
Takes the comprehensive fault simulation testbed of Spectra Quest as an object, the finite element model of rotor-rolling bearing-base system is constructed by finite element analysis software ANSYS. Modes of rotor-rolling bearing system and rotor-rolling bearing-base system are analysed, the influence of base is researched by comparing their natural frequency and vibration mode. The influence of bearing rigidity to system mode is studied by comparing the natural frequency of rotor-rolling bearing-base system with different bearing rigidity.
\end{abstract}

\section{Introduction}

The modal analysis of rotor-rolling bearing-base system is of great importance to study the abnormal vibration of large high speed rotors and maintain the stable and safe operation of units. As part of dynamic analysis and an important branch of vibration engineering theory, modal analysis is a powerful tool for product structure designing and performance evaluation.

However, it is impossible to understand the overall performance of a structure by analyzing its individual parts in a modal analysis, since the parameters of each part joint affect the overall dynamic characteristics. Therefore, in order to fully understand the dynamic characteristics of complex structures, it is necessary to conduct modal and dynamic analysis to the entire structure. This thesis carries out modal analysis on rotating parts and the whole system respectively, and compares the influence of different bearing stiffness on the inherent characteristics of rotating parts.

\section{The Theoretical Basis for Modal Analysis}

In a modal analysis, the motion of any system can be described by a kinetic differential equation of a system with $\mathrm{N}$ degrees of freedom.

$$
\mathbf{M} \ddot{X}+\mathbf{C} \dot{X}+\mathbf{K} X=0
$$

Suppose the solution to equation (1) is

$$
X=\varphi e^{\lambda t}
$$

$\varphi$ is a pending often sequence vector and $\lambda$ is complex frequency

By plugging (2) into (1), the equation of vibration characteristics is derived:

$$
\left(\lambda^{2} \mathbf{M}+\lambda \mathbf{C}+\mathbf{K}\right) \varphi=0
$$

By working out the solution to (3), we can get the natural frequency and vibration mode of the system. For a system with $N$ degrees of freedom, it has $N$ natural frequencies. The eigenvector corresponding with the natural frequency is referred to as the natural mode or mode shape. With the mode shape corresponding to the structural perturbations figure, the structural vibration shape at any moment is the linear combination of the mode.

\section{The Establishment of the Finite Model}

The parts drawings of the rotor, rolling bearing, bearing pedestal and supporting pedestal are respectively established in the Pro/E, and according to the assembly design, the parts are assembled into an assembly, which is then imported into ANSYS. 
The BEAM188 unit is adopted to simulate the rotor and the SOLID95 unit to simulate the base, supporting and bearing pedestal. As the rolling element of bearings is elastic in actual conditions, the bearings can be simplified as the model in Fig. 1 suggests, namely, the balls can be replaced by four spring-dampers, one side of which is connected with the bearing pedestal and the other side with the rotor. In addition, the COMBIN14 unit in ANSYS is specialized for the simulation of spring-dampers, thus this unit can simplify the bearings.

With the use of screws, the rotor-rolling bearing-base system is fixed onto the floor and the bearing pedestal is installed on the base. The rotor is driven by the electric motor. According to actual conditions in installing, the degree of freedom at all directions in the base supporting area should be strictly restricted so as to keep the whole system fixed. The axial displacement of the rotor also should be constrained to avoid the axial sliding of the rotor, and the rotor's end that is connected with the motor should be allowed degree of freedom to rotate axially. Fig. 2 is the boundary constraint finite model of the rotor-rolling bearing-base system.

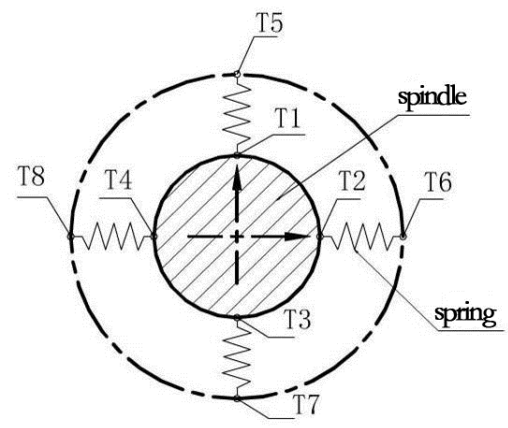

Fig. 1 Simplified version of the rolling bearing

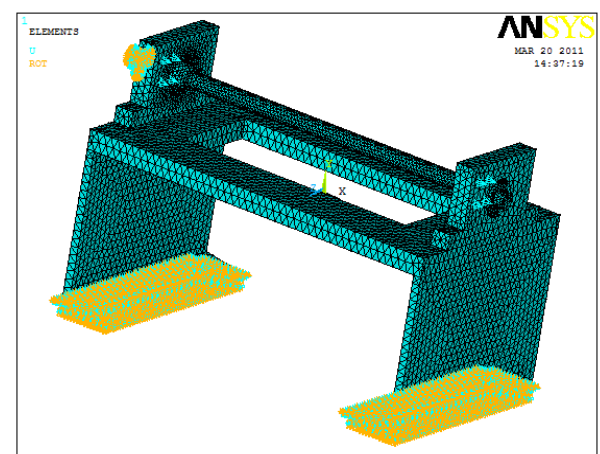

Fig. 2 Boundary constraint finite model of the rotor-rolling bearing-base system

\section{Modal Analysis}

Modal Analysis of the Rotor-Rolling Bearing System. Firstly, conduct a modal analysis independently of the rotating parts in the rotor-rolling bearing system. In the simplified version of the rolling bearing, four rotor and spring connection nodes (T1, T2, T3, T4) are given translational constraints in order to control the rotor's axial displacement, while the other ends of the springs (T5, T6, T7, T8) are completely fixed.

Use the COMBIN14 unit to mesh the rolling bearing, ignore the influence of damping and regard rolling bearing stiffness as a constant free of the bearing's overload and rotating speed. Fig. 3 is the rotor-rolling bearing finite model with constraint conditions, and Fig. 4 shows the constraint situation after substituting the spring-dampers on the rolling bearing.

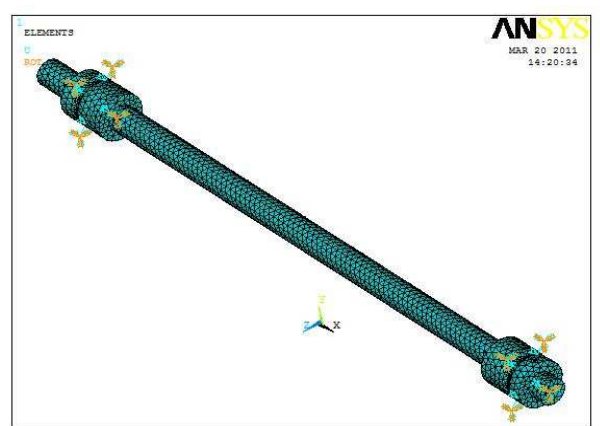

Fig. 3 Rotor-rolling bearing finite model with constraint conditions

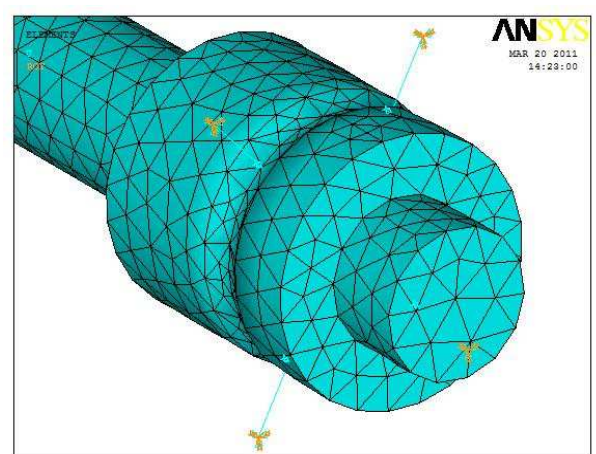

Fig. 4 Simplified rolling bearing finite model

A structure's vibration can be described by the linear combination of various natural vibration modes, among which low-order natural vibration mode plays a decisive role in determining the structure's dynamic characteristics, more influential than the high-order natural vibration mode. Block Lanczos is adopted to gain the first eight natural frequencies and vibration modes. Table 1 is the first eight natural frequencies and Fig. 5 is the vibration modes of the rotor-rolling bearing system. 
Table 1 The First eight modal frequency of the rotor-rolling bearing system

\begin{tabular}{ccccc}
\hline Order & 1 & 2 & 3 & 4 \\
\hline Natural Frequency/Hz & 447.30 & 534.31 & 689.83 & 910.21 \\
\hline Order & 5 & 6 & 7 & 8 \\
\hline Natural Frequency $/ \mathrm{Hz}$ & 996.77 & 1200.5 & 1876.9 & 2033.1 \\
\hline
\end{tabular}

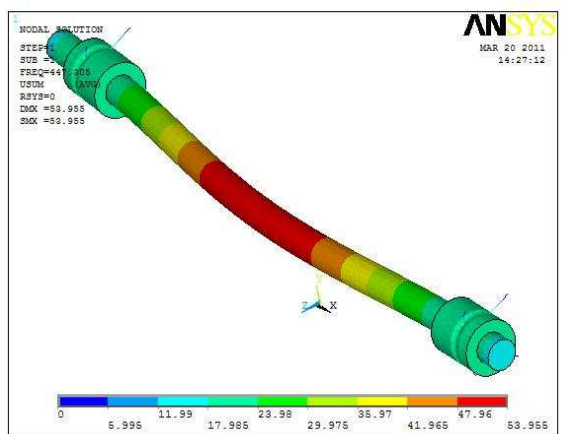

The first mode

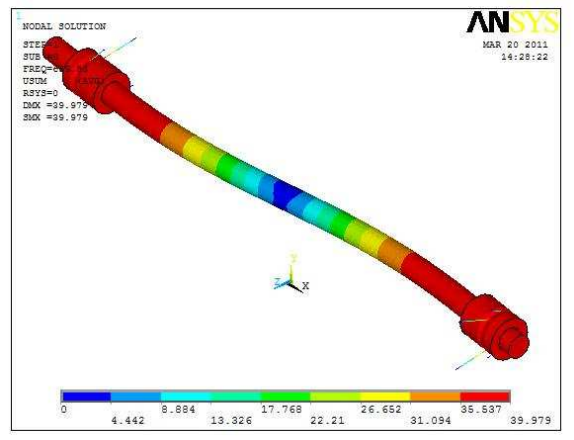

The third mode

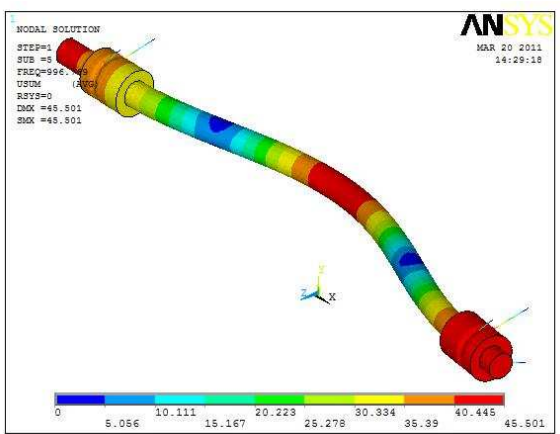

The fifth mode

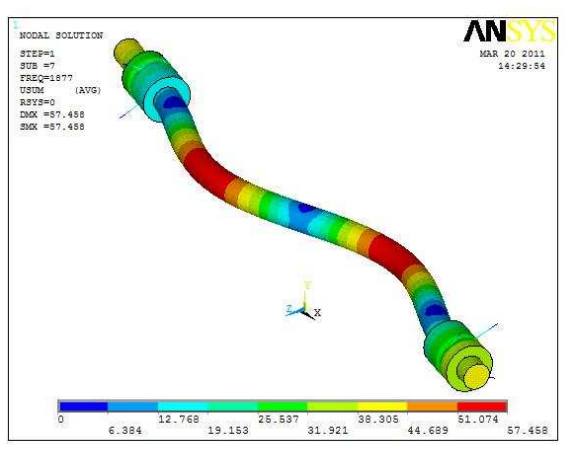

The seventh mode

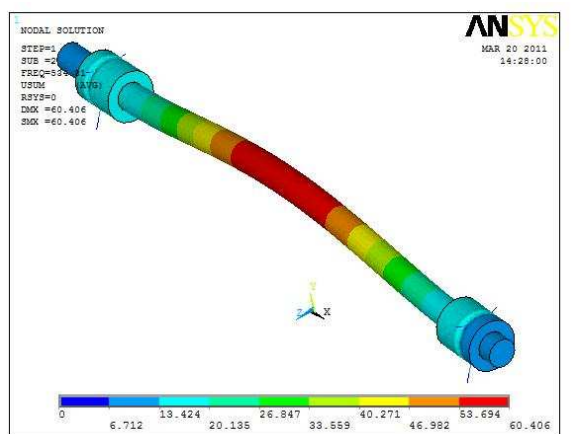

The second mode

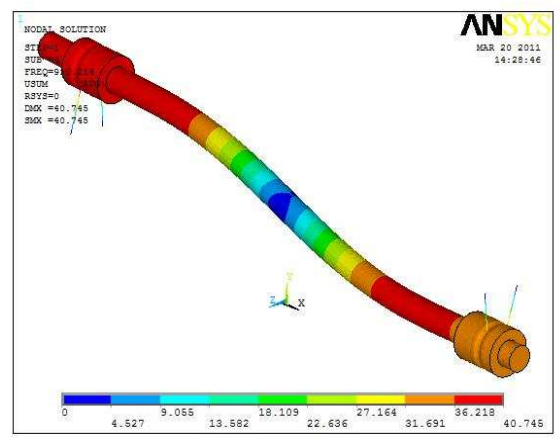

The fourth mode

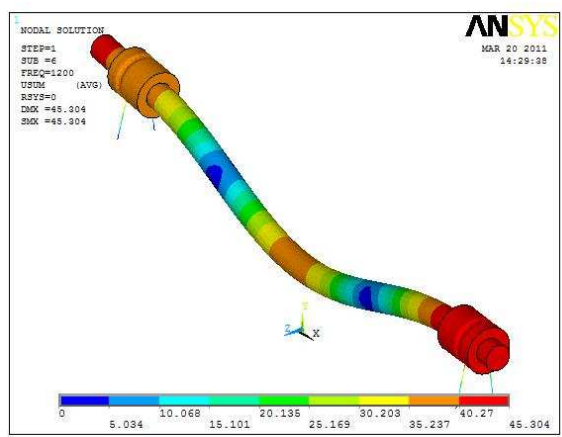

The sixth mode

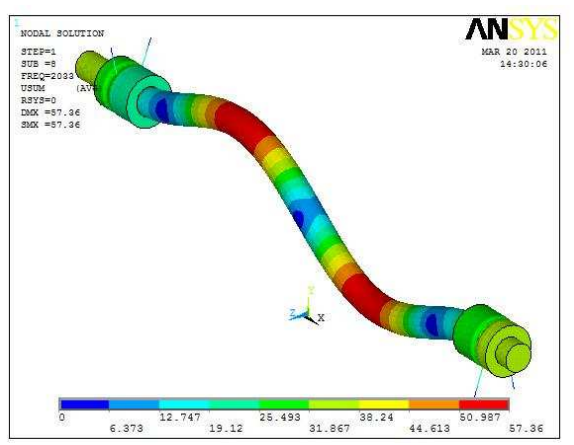

The eighth mode

Fig. 5 Vibration mode of the rotor-rolling bearing system

The vibration mode value is a relative value (relative displacement value) and suggests the relative vibration value of every spot at a certain natural frequency. It reflects the vibration transmission situation at various natural frequencies instead of the actual vibration value. 
The results reveal that the rotor undergoes flexural vibration for the first and second mode. The middle part of the rotor has the highest amplitude and may easily break. While for the third, the fourth, the fifth and the sixth mode, the bearing has the highest amplitude and may finally break.

Modal Analysis of the Rotor-rolling bearing-base System. In order to fully understand the dynamic characteristics of complex structures, it is necessary to consider the flexibility of the base, conduct modal and dynamic analysis to the entire structure. The first eight natural frequencies and vibration modes of rotor-rolling bearing-base system are gained inTable 2 and Fig. 6 suggests.

Table 2 The first eight modal frequencies of the rotor-rolling bearing-base system

\begin{tabular}{ccccc}
\hline Order & 1 & 2 & 3 & 4 \\
\hline Natural frequency/Hz & 166.21 & 368.15 & 482.65 & 563.86 \\
\hline Order & 5 & 6 & 7 & 8 \\
\hline Natural frequency $/ \mathrm{Hz}$ & 630.85 & 839.90 & 991.01 & 1049.3 \\
\hline
\end{tabular}

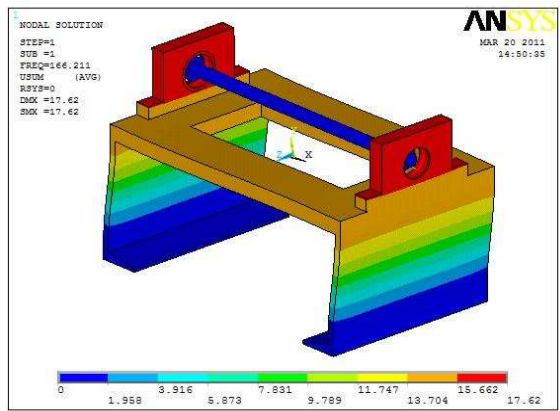

The first mode

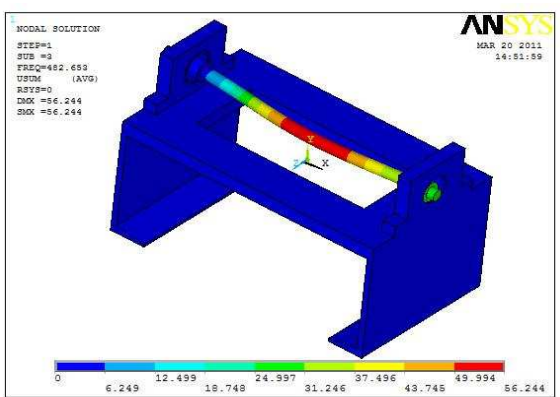

The third mode

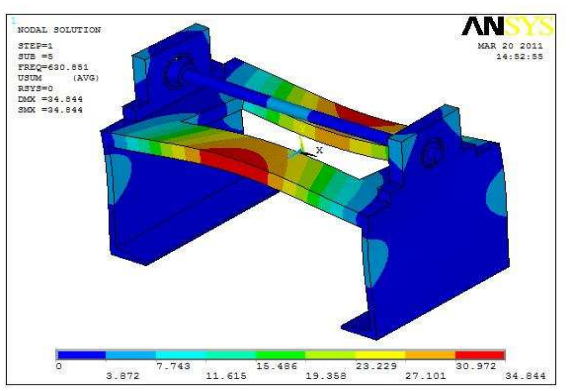

The fifth mode

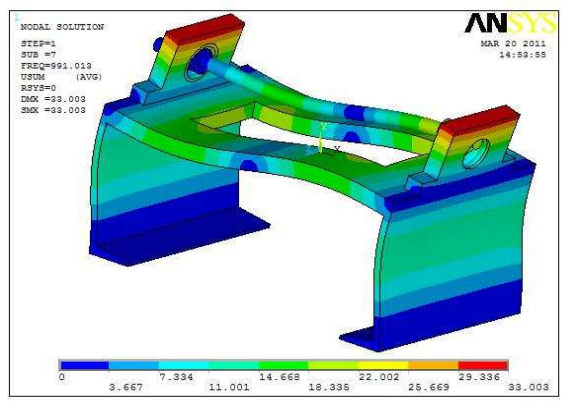

The seventh mode

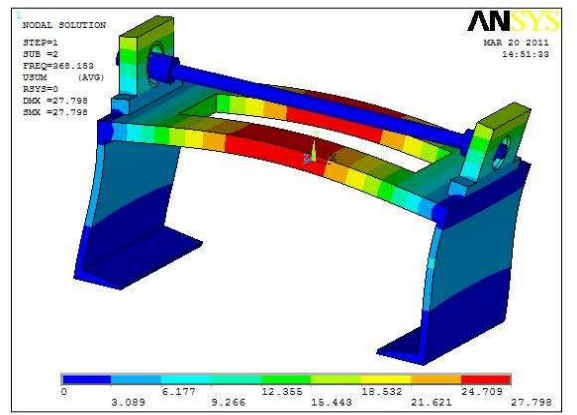

The second mode

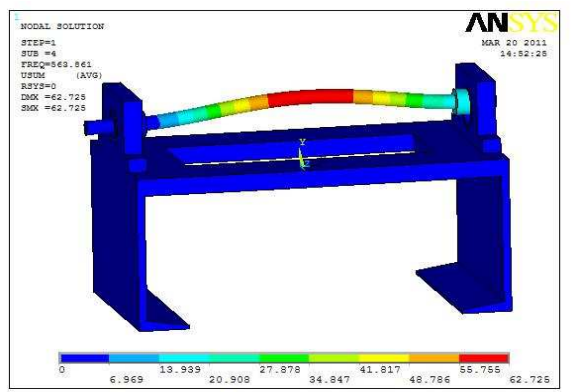

The fourth mode

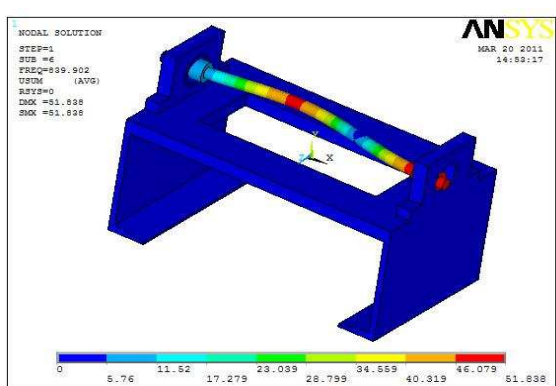

The sixth mode

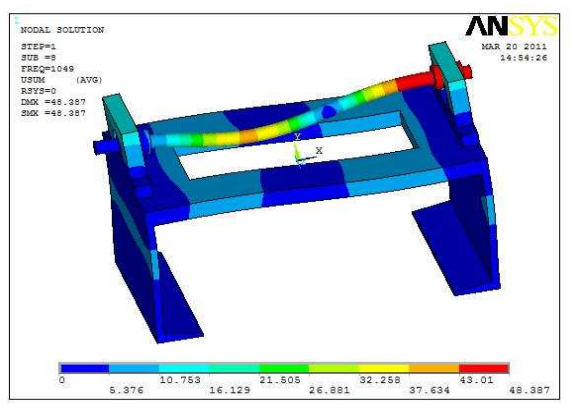

The eighth mode

Fig. 6 Vibration mode of the rotor-rolling bearing-base system 
It can be seen from the calculations that the natural frequency of the rotor-rolling bearing-base system is lower than that of the rotor-rolling bearing system. Among them, the third, the fourth and the sixth modes are local deformation modals of the rotor; the first and second modes show the overall modals of the base system; the fifth, the seventh and the eighth modes are deformation modals when the entire base system and the rotor-rolling bearing subsystem are interacting with each other. In the base system, the first mode shows the highest vibration amplitude of the bearing pedestal and thus the bearing will be affected; the second and fifth modes demonstrate the highest vibration amplitude of the base; the third and the fourth modes feature the rotor's rotation.

Influence of Bearing Stiffness on the Rotor-rolling bearing-base System Modal. In conducting modal analysis of the rotor-rolling bearing-base system, bearing stiffness exerts much influence on the modal of the whole system. This section analyzes the influence of different bearing stiffness on the inherent characteristics of the whole system, and comprehensively researches into the system's natural frequency with different bearing stiffness by selecting the first five modals, as shown in Table 3 and Fig. 7 demonstrates the influence of different bearing stiffness on the inherent characteristics of the whole system. It can be seen from the figure that the system's natural frequency increases when bearing stiffness increases.

Table 3 System natural characteristics of different bearing stiffness (Unit: $\mathrm{Hz}$ )

\begin{tabular}{lcccccc}
\hline & $1000 \mathrm{~N} / \mathrm{mm}$ & $10^{4} \mathrm{~N} / \mathrm{mm}$ & $10^{5} \mathrm{~N} / \mathrm{mm}$ & $10^{6} \mathrm{~N} / \mathrm{mm}$ & $10^{7} \mathrm{~N} / \mathrm{mm}$ & Rigid connection \\
\hline Mode 1 & 166.2 & 166.23 & 166.32 & 166.35 & 166.36 & 166.36 \\
Mode 2 & 315.93 & 368.99 & 371.96 & 373.02 & 373.15 & 373.17 \\
Mode 3 & 367.13 & 541.61 & 576.89 & 581.2 & 581.67 & 581.72 \\
Mode 4 & 408.08 & 599.84 & 624.18 & 628.4 & 628.9 & 628.95 \\
Mode 5 & 630.36 & 631.57 & 634.37 & 635.49 & 635.64 & 635.66 \\
\hline
\end{tabular}

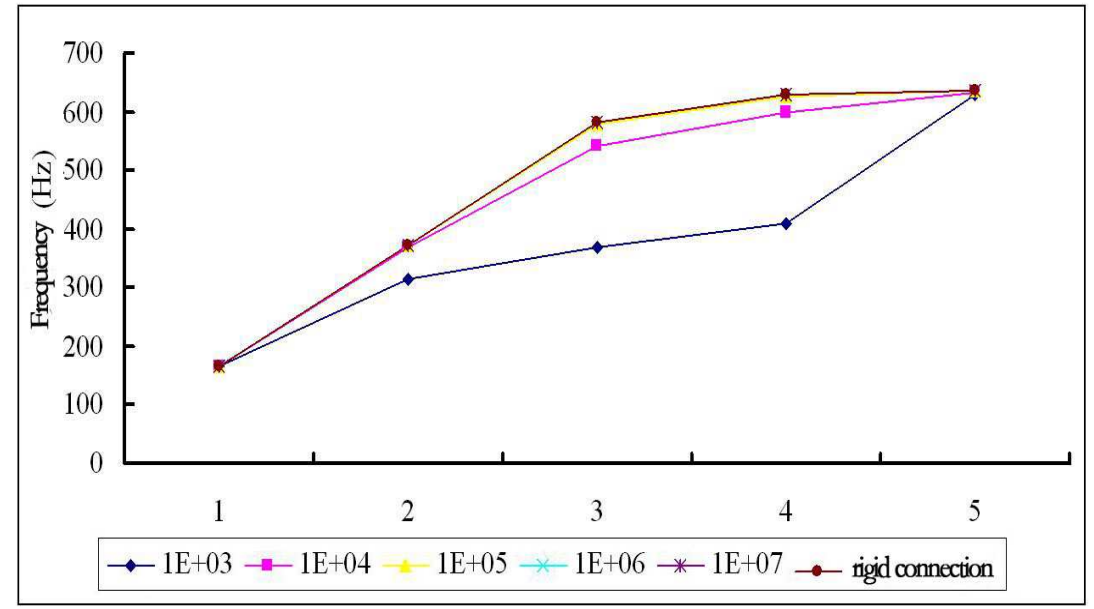

Fig. 7 The system's inherent characteristics with different bearing stiffness

By analyzing the relation between natural frequencies of different orders and bearing stiffness, it can be known that bearing stiffness greatly affects the whole system's natural frequency. The larger the bearing stiffness is, the higher the low-order natural frequency is. Therefore, without changing the structure of the whole system, it is feasible to adjust the critical speed and the system's natural frequency by changing bearing stiffness.

\section{Conclusion}

This thesis conducts modal analyses of the rotor-rolling bearing system and the rotor-rolling bearing-base system respectively with the aid of the ANSYS software and the Block Lanczos. By taking the system's bearing flexibility into account in the dynamic response analysis, the results are more objective. This thesis reaches the following conclusions: 
1. Given the consideration of the base's flexibility factors, the modal of the rotor-rolling bearing-base system is lower than that of the rotor-rolling bearing system. Among the first eight vibration modes of the whole system, the third, the fourth and the sixth are the rotor's local deformation modals.

2. Rolling bearing stiffness can greatly affect the modal of the rotor-rolling bearing-base system. Without changing the structure of the whole system, it is feasible to adjust bearing stiffness by changing the pretightening force of the rolling bearing and further alters the whole system's modal.

\section{Acknowledgement}

Financial support from Aid program for Science and Technology Innovative Research Team in Higher Educational Institutions of Hunan Province, the CEEUSRO special plan of Hunan province (2010XK6066), The Industrial Cultivation Program of Scientific and Technological Achievements in Higher Educational Institutions of Hunan Province (10CY008), New Century Excellent Talents (NCET-08-0677), Natural Science Foundation of Hunan Province Key Project (09JJ8005), are gratefully acknowledged.

\section{References}

[1] L. Yu, H. Liu, Dynamics of Bearing-Rotor System, Xi'an Jiaotong University Press, 2001.

[2]Z.Y. Shang, Nonlinear Dynamics Analysis of Rotor-Bearing System, Harbin Industrial University, 2006.

[3] X.H. Zhu, Z.X. Yu Zhixiang, The Selected Senior Project Examples of ANSYS Finite Analysis, The Electronics Industry Press, 2005.

[4] C.M. Song, N. Zhao, Z.W. Zhang, Research of Static and Dynamic Characteristics of High-speed Electic Spindle Based on Ansys, J. Coal Mine Machinery, 2007, 28(4): 58-60.

[5] A.L. Hu, The Finite Analysis of High-speed Rotor Axis, Guangdong University of Technology, 2004.

[6] L. Liu, G. Zhang, G. Gao, Z.F. Zhao, Finite Element Analysis on Cylindrical Roller Bearing Based on ANSYS,J. Bearing, 2006, 12: 8-12.

[7] B.H. Yao, X.J. Li, T. Zhang, Finite Element Modal Analysis of Cracked Rotor-bearing-base System, J. Journal of Hunan University of Science \& Technology(Natural Science Edition), 2010, 25(3): 32-35. 